\title{
L'epidemiologia dell'arteriopatia periferica nell'insufficienza renale cronica
}

\author{
Paola Brescia
}

Struttura Complessa di Nefrologia e Dialisi, A.O. Carlo Poma, Mantova

\begin{abstract}
EPIDEMIOLOGY OF PERIPHERAL ARTERY DISEASE IN CKD PATIENTS
Abstract. Peripheral artery disease (PAD) is underdiagnosed, undertreated, and poorly understood by the medical community. Yet, PAD is a strong predictor of coronary artery disease and a risk factor for mortality in the general population. This is of particular interest to nephrologists because the prevalence of PAD appears to be much higher among patients with end stage renal disease (ESRD) than in the general population. This is explained by the association between traditional and uremia-related risk factors. In fact, kidney disease is an independent risk factor for the development of PAD, with risk increasing with worsening kidney function. The current epidemiology in ESRD patients is here reviewed.
\end{abstract}

Key words: Peripheral artery disease, Chronic kidney disease, Epidemiology, Ankle-brachial index

Conflict of interest: None.

Financial support: None.

Ricevuto: 10 Aprile 2013; Accettato: 29 Maggio 2013

L'Arteriopatia Periferica (AP) è una condizione di aterosclerosi caratterizzata da un'ischemia progressiva della muscolatura scheletrica e della cute delle estremità. Due sono le forme cliniche in cui può manifestarsi:

la Claudicatio Intermittens, ovvero l'insorgenza di dolore agli arti inferiori dopo aver percorso un perimetro di marcia ben definito;

la Critical Limb Ischemia, ossia la presenza di dolore a riposo, persistente, che dura da almeno 15 giorni, associato o meno alla presenza di lesioni ischemiche, la cui evoluzione, in caso di mancata guarigione, porta all'amputazione dell'arto interessato a vari livelli a seconda della gravità delle lesioni.

L'AP nei pazienti affetti da CKD presenta una prevalenza e un'incidenza molto più elevate rispetto alla popolazione generale. Quando, infatti, questa categoria di pazienti viene messa a confronto con individui con funzione renale normale, essa mostra un rischio più elevato di sviluppare aterosclerosi generalizzata e malattia cardiovascolare (1). Questa differenza la si nota anche quando vengono messi a confronto i classici fattori di rischio cardiovascolare (età, sesso maschile, tabagismo, diabete, dislipidemia, ipertensione e IRC), associati, nella popolazione generale, a un aumentato rischio di AP. La malattia renale è un fattore di rischio indipendente per lo sviluppo di AP e il rischio aumenta con il peggioramento della funzione renale (1). Questo è dovuto alla combinazione di fattori di rischio tradizionali con fattori di rischio non tradizionali quali lo stato di infiammazione cronica dei pazienti uremici, lo stress ossidativo causa di ipoalbuminemia, elevati livelli di omocisteinemia, elevati livelli di lipoproteina (a), l'alterazione del metabolismo calcio-fosforo e l'iperparatiroidismo. A tutto ciò si sommano gli effetti di tutte le altre condizioni morbose con le quali gli uremici convivono, OSTEOARTRITI di varia natura, INFEZIONI ricorrenti, NEUROPATIE diabetiche e non, che diminuiscono le probabilità di fare diagnosi precoce di AP, cioè allo stadio di claudicatio intermittens, quando le terapie tradizionali a disposizione eviterebbero amputazioni devastanti e invalidanti.

L'analisi epidemiologica dell'AP, valutando la sua distribuzione e la sua frequenza e analizzandone le cause, il decorso e le conseguenze, conferma la severità del quadro clinico nei pazienti uremici. L'AP nei pazienti affetti da CKD presenta una prevalenza, l'insieme di tutti $\mathrm{i}$ casi esistenti in una popolazione in un momento ben determinato, e un'incidenza, il verificarsi di nuovi casi, molto più elevate che nella popolazione generale. Quando, infatti, questa categoria di soggetti viene messa a confronto con individui con funzione renale normale, essa presenta un rischio più elevato di sviluppare aterosclerosi generalizzata e malattia cardiovascolare (1). Diversi studi hanno affrontato l'argomento AP nei nefropatici dal punto di vista epidemiologico:

- ARIC, Aterosclerosis Risk in Communities Study, studio prospettico eseguito su 15792 pazienti tra i 45 e i 64 anni tra 


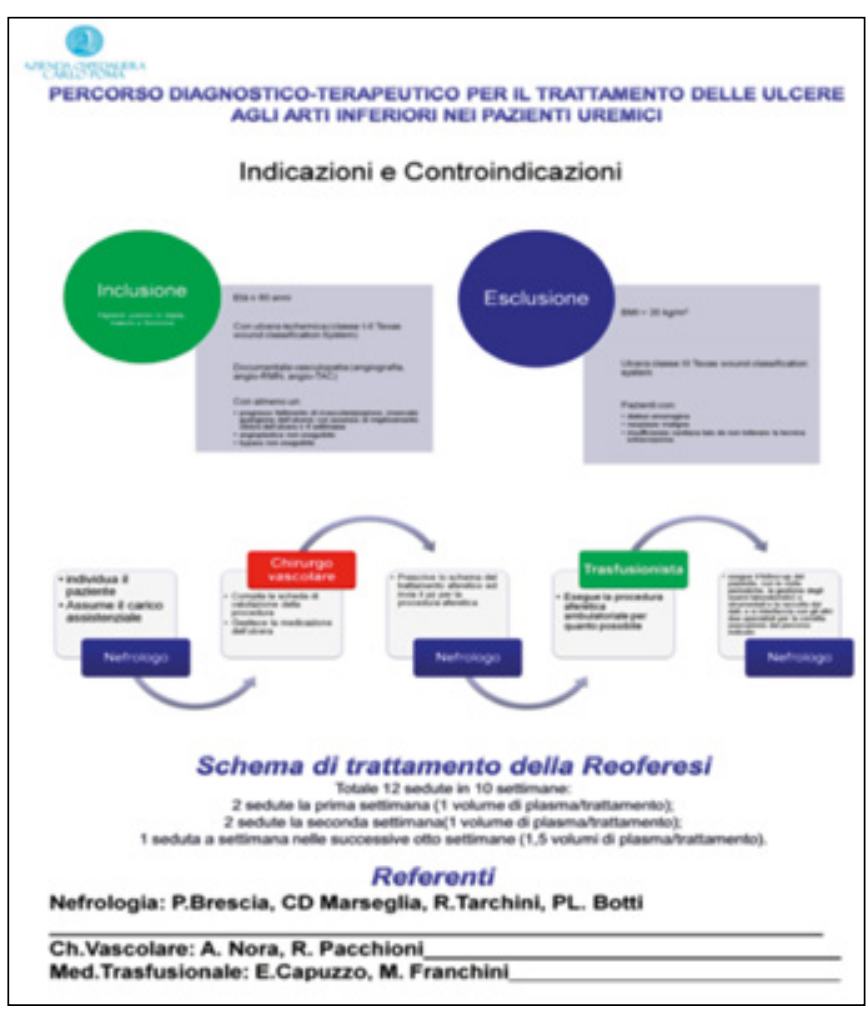

Fig. 1 - Flow chart: iter diagnostico terapeutico per il trattamento delle ulcere agli arti inferiori nei pazienti uremici.

il 1987 e il 1989, con un follow-up periodico fino al 1998, sulla storia naturale dell'aterosclerosi e della malattia cardiovascolare, mostra come i pazienti con IRC siano a più elevato rischio di AP. La stima dell'incidenza annuale, calcolata su 1000 persone, è di 4.7 nei soggetti con normofunzione renale, di 4.9 nei soggetti con IRC di media entità e, addirittura, di 8.6, quando viene calcolata su soggetti con IRC avanzata.

- USRDS, United States Renal Data System Dialysis, Morbidity and Mortality Study, un sistema di raccolta dati adoperato negli Stati Uniti, calcola la prevalenza di AP su un campione di 35438 pazienti in dialisi, riportando una percentuale pari al $45.9 \%$.

- HEMO, Hemodialysis Study, studio eseguito su 936 pazienti in $\mathrm{HD}$, riporta una prevalenza di AP del $23 \%$ in un campione di dializzati.

- Stime di prevalenza di AP eseguite su trapiantati di rene hanno segnalato una percentuale compresa tra il $15 \%$ e il $30 \%$. Questi valori raggiungevano punte del $46 \%$ nel doppio trapianto rene-pancreas.

C'è da tenere conto del fatto che queste determinazioni sono state realizzate in soggetti dove la diagnosi di AP era fatta con il solo ausilio dei sintomi, ossia del quadro clinico, il che significa che questi dati sottostimano la reale percentuale di AP nei dializzati (3). Quando, infatti, si considerano studi che valutano la prevalenza di AP in pazienti con ESRD, utilizzando test diagnostici non invasivi, si raggiungono percentuali comprese tra il $16 \%$ e il $38 \%$, a seconda della specifica popolazione in esame, e, in generale, la prevalenza di AP tra i dializzati risulta doppia se si

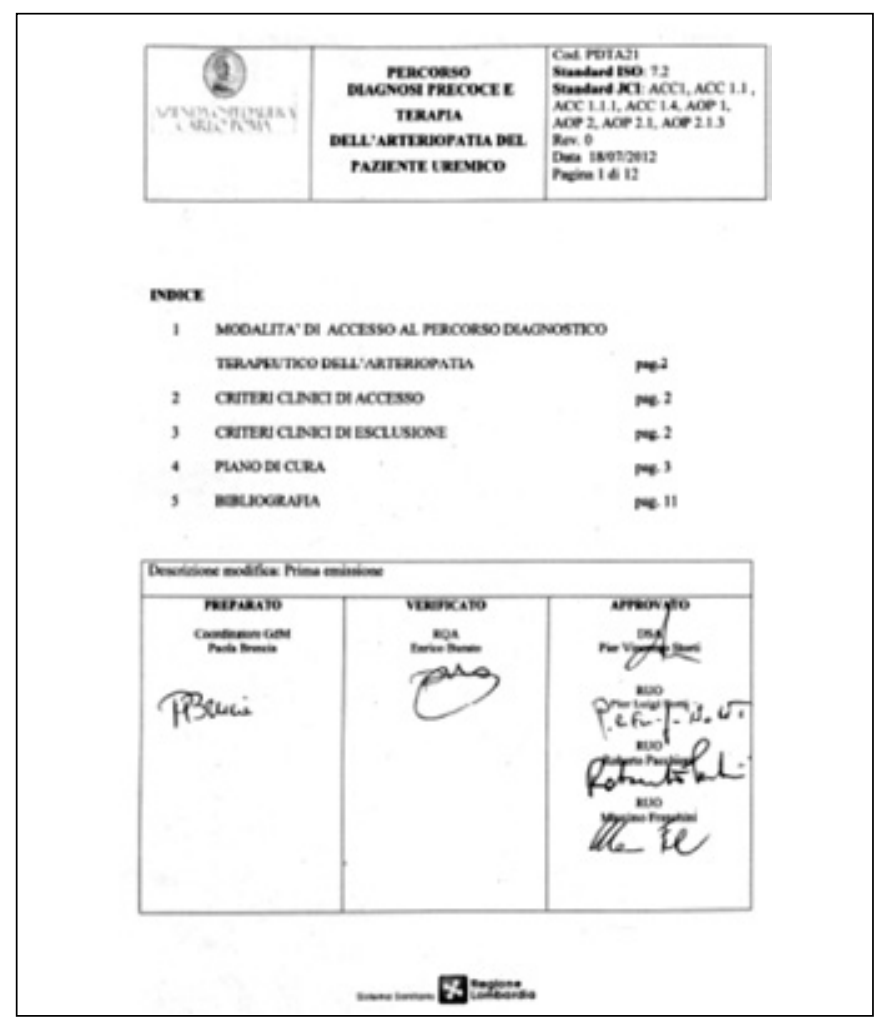

Fig. 2 - I pagina del plico ufficiale dell'iter diagnostico terapeutico controfirmato da tutti gli specialisti.

valuta attraverso l'ABI, ankle-brachial index: rapporto tra la PA sistolica misurata a livello dell'arteria tibiale e la PA sistolica misurata a livello dell'arteria brachiale, rispetto a quella valutata considerando solo la storia clinica dei pazienti (3).

- NHANES 1999-2000, National Health and Nutrition Examination Survey, ha chiaramente dimostrato un aumentato rischio di presenza di AP associato con il grado di funzione renale residua. In altre parole, minore è la funzione renale residua maggiore è il rischio di sviluppare AP (5).

- DOPPS, Peripheral Arterial Disease in Patients With EndStage Renal Disease; questo studio prospettico, internazionale e osservazionale, eseguito su 29873 pazienti in emodialisi, ha dimostrato come l'AP sia particolarmente frequente nei dializzati e sia associata a un aumentato rischio di complicanze e mortalità cardiovascolari, oltre che a un'aumentata percentuale di ospedalizzazione e $\mathrm{a}$ una bassa qualità di vita (6).

Alla luce di questi dati, De Loache et al. (4) hanno focalizzato i punti fondamentali su cui il nefrologo deve porre attenzione per una corretta diagnosi e un'adeguata terapia di quei nefropatici che dimostrano la presenza di AP.

In cima alla lista troviamo i fattori di rischio distinti in modificabili, diabete, tabagismo, $\mathrm{CKD} / \mathrm{ESRD}$, dislipidemia e ipertensione, e immodificabili, sesso maschile, razza nera ed età. Seguono i segni e i sintomi della malattia: claudicatio, dolore a riposo, ulcere e gangrena. Vengono, quindi, consigliati 2 test non invasivi che consentirebbero di diagnosticare la malattia in assenza di sintomi: ABI (Ankle-Brachial-Index) e TBI (Toe- 
Brachial-Index). In aggiunta a questi ultimi, andrebbero eseguite una risonanza magnetica, una tomografia computerizzata o un'angiografia, per una migliore definizione diagnostica.

Seguono consigli sulla terapia medica tradizionale, sullo stile di vita e, infine, su possibili correzioni chirurgiche della malattia. In conclusione, vista l'alta frequenza di AP nella popolazione uremica, una corretta condotta di comportamento prevede una monitorizzazione attenta della patologia, con una diagnosi quanto più possibile precoce e una messa in atto di tutte le possibili manovre di prevenzione, al fine di scongiurare il rischio di amputazione e morte di questi soggetti.

A questo scopo, nell'Azienda Ospedaliera di Mantova, abbiamo messo a punto un percorso diagnostico terapeutico per la diagnosi precoce e il trattamento delle ulcere agli arti inferiori nei pazienti uremici (Fig. 1). Il percorso si avvale di una competenza multidisciplinare, a conferma della necessità di un coinvolgimento di più specialisti per il trattamento dell'AP (Fig. 2). Il fine ultimo di tale team è la realizzazione di un approccio terapeutico integrato, che comprenda anche procedure di aferesi terapeutica per la correzione dell'ulcera.

\section{Riassunto}

L'arteriopatia periferica (AP) non è al momento adeguatamente diagnosticata e trattata dalla comunità medica. Eppure, l'AP rappresenta un potente predittore di coronaropatia e un fattore di rischio per mortalità nella popolazione generale. Questo aspetto dovrebbe essere di particolare interesse per i nefrologi, dato che la prevalenza di AP è sicuramente più elevata nei pazienti uremici che nella popolazione generale. La spiegazione del fenomeno è legata all'associazione, in questi pazienti, dei tradizionali fattori di rischio con quelli specifici della condizione uremica. Infatti, la malattia renale di per sé è un fattore di rischio indipendente per lo sviluppo di AP, con un rischio che si rafforza al progressivo declino della funzione renale. L'attuale situazione epidemiologica dell'AP nei pazienti uremici è oggetto di questa revisione.

Parole chiave: Arteriopatia periferica, Insufficienza renale cronica, Epidemiologia, Indice caviglia-braccio

Dichiarazione di conflitto di interessi: L'Autore dichiara di non avere conflitto di interessi.

Contributi economici degli autori: L'Autore dichiara di non aver ricevuto sponsorizzazioni economiche per la preparazione dell'articolo.

Indirizzo degli Autori:

Dr. Paola Brescia

Struttura Complessa di Nefrologia e Dialisi

A.O. Carlo Poma

Via Lago Paiolo 10

46100 Mantova

paola.brescia@aopoma.it

\section{Bibliografia}

1. Garimella PS, Hart PD, O'Hare A, et al. Peripheral Artery Disease and CKD: A Focus on PAD as a Critical Component of CKD Care. Am J Kidney Dis 2012; 60 (4): 641-54.

2. Wattanakit K, Folson AR, Selvin E, et al. Kidney Function And Risk of Peripheral Arterial Disease: Results from the Atherosclerosis Risk in Communities (ARIC) Study. J Am Soc Nephrol 2007; 18 (2): 629-36.

3. O'Hare A, Johansen K. Lower Estremity Peripheral Arterial Disease Among Patients with End-Stage Renal Disease. J Am Soc
Nephrol 2001; 12 (12): 2838-47.

4. De Loach SS, Mohler E. Peripheral Arterial Disease: A Guide for Nephrologists. Clin J Am Soc Nephrol 2007; 2 (4): 839-46.

5. Selvin E, Kottgen A, Coresh J. Kidney Function Estimated from Serum Creatine and Cystatin C and Peripheral Arterial Disease in Nhanes 1999-2002. Eur Heart J 2009; 30 (15): 1918-25.

6. Rajagopalan S, Dellegrottaglie S, Furniss AL, et al. Peripheral Arterial Disease in Patients With End-Stage Renal Disease: Observations From the Dialysis Outcomes and Practice Patterns Study (DOPPS). Circulation 2006; 114: 1914-22. 